\title{
Non-local Multi-continua Upscaling for Flows in Heterogeneous Fractured Media
}

\author{
Eric T. Chung* Yalchin Efendiev ${ }^{\dagger} \quad$ Wing Tat Leung ${ }^{\ddagger}$ \\ Yating Wang ${ }^{\S} \quad$ Maria Vasilyeva
}

August 29, 2017

\begin{abstract}
In this paper, we propose a rigorous and accurate non-local (in the oversampled region) upscaling framework based on some recently developed multiscale methods [10. Our proposed method consists of identifying multi-continua parameters via local basis functions and constructing non-local (in the oversampled region) transfer and effective properties. To achieve this, we significantly modify our recent work proposed within Generalized Multiscale Finite Element Method (GMsFEM) in [10] and derive appropriate local problems in oversampled regions once we identify important modes representing each continua. We use piecewise constant functions in each fracture network and in the matrix to write an upscaled equation. Thus, the resulting upscaled equation is of minimal size and the unknowns are average pressures in the fractures and the matrix. We note that the use of non-local upscaled model for porous media flows is not new, e.g., in [14, the authors derive non-local approach. Our main contribution is identifying appropriate local problems together with local spectral modes to represent each continua. The model problem for fractures assumes that one can identify fracture networks. The resulting non-local equation (restricted to the oversampling region, which is several times larger compared to the target coarse block) has the same form as 14. with much smaller local regions. We present numerical results, which show that the proposed approach can provide good accuracy.
\end{abstract}

*Department of Mathematics, The Chinese University of Hong Kong, Shatin, New Territories, Hong Kong SAR, China (tschung@math.cuhk.edu.hk)

${ }^{\dagger}$ Department of Mathematics \& Institute for Scientific Computation (ISC), Texas A\&M University, College Station, Texas, USA (efendiev@math.tamu.edu)

‡Department of Mathematics, Texas A\&M University, College Station, TX 77843, USA (leungwt@math . tamu . edu)

$\S^{\S}$ Department of Mathematics, Texas A\&M University, College Station, TX 77843, USA (wytgloria@math.tamu.edu)

I Institute for Scientific Computation, Texas A\&M University, College Station, TX, USA \& Department of Computational Technologies, North-Eastern Federal University, Yakutsk, Republic of Sakha (Yakutia), Russia (vasilyevadotmdotv@gmail.com) 


\section{Introduction}

\subsection{Flow-based upscaling methods}

Because of the level of detail in geological formations, some type of coarsening or upscaling is typically performed. In upscaling methods, media properties are upscaled and effective properties are computed for each coarse block [2, 18, 12, [15, 4, 40, 17]. Computing effective properties involves solving local problems and equating the averages of local integrated quantities. For example, computing upscaled permeabilities in reservoir simulation is typically based on equating average fluxes between the local fine-grid solves and the coarse-grid solves. This equality allows computing the effective permeability fields.

In a more general upscaling setup, a multiple continua approach 8 is needed. In this approach, several effective properties are computed for each coarse block in addition to modeling the transfer terms. This computation involves evaluating both effective properties and transfer coefficients between different continua. The computations are performed locally.

\subsection{Multiscale methods and their relation}

Similar to upscaling methods, many authors have recently studied multiscale methods. In multiscale methods [27, 25, 30, 33, 21, [11, 28, 3, 29, 35, 31, 34, 20. 7, 24, 26, 9, instead of computing the effective properties, one computes multiscale basis functions. For single-phase upscaling, a multiscale basis function for each coarse node is computed via local solutions. These basis functions are further coupled via a global formulation of the equation. This approach is implemented within Multiscale Finite Element Method (MsFEM) and other multiscale methods [27, 28, 20, 7, 1, 37, 36, 5, 6].

To generalize this approach to more complex heterogeneities and the multicontinua case, Generalized Multiscale Finite Element Method (GMsFEM) is proposed [20, 7, 9, 10]. GMsFEM proposes a systematic approach to compute multiple basis functions. This approach starts with a space of snapshots, where one performs local spectral decomposition to compute multiscale basis functions. Adaptivity can be used to select basis functions in different regions. Each multiscale basis function represents a continua as discussed in [8] and there is no need for coupling terms between these continua. The basis functions for each continua are automatically identified.

The GMsFEM approach has been used jointly with localization ideas in [10, where the authors propose Constraint Energy Minimizing GMsFEM. In this approach, oversampling regions are used to compute the multiscale basis functions. This construction takes into account spectral basis functions to localize the computations. The localization is restricted to $\log (H)$ layers and depends on the contrast, which can be reduced using snapshot functions. Moreover, it was shown that the approach converges independent of the contrast and the convergence is linear with respect to the coarse mesh size. More precisely, the convergence is proportional to $H / \Lambda^{1 / 2}$, where $\Lambda$ is the smallest eigenvalue 
that the corresponding eigenfunction is not included in the coarse space. Note that basis functions associated to fractures correspond to very small eigenvalues. The goal of this paper is to modify this framework in an appropriate way that is more suitable for flow-based upscaling and re-cast it as non-local upscaling.

\subsection{This paper}

To modify the multiscale approach presented in [10, we first assume that one knows each separate fracture network within a coarse-grid block. This is one of the drawbacks of our method; however, such cases occur in many applications. Next, we follow a general concept of spectral basis functions and simply define constant functions in each fracture network and the matrix. Because the fracture has zero width, this procedure needs to be carefully formulated, which is done in the paper.

Secondly, we solve local problems in the oversampled region subject to the constraint that the local problems vanish in fractures and the matrix. This condition is imposed as a constraint to the local problem and important for the localization. The local problems formulated for each continua (either fracture network or the matrix phase) simply minimize the local energy subject to the constraint that the local solution "vanishes" in other continua except the one for which it is formulated for. More precisely, for the continua $i$ in a block $K$, we minimize the local oversampled problem such that it is orthogonal to all continua except $i$ and an appropriate inner product with the continua $i$ is 1 .

It is important to note that the localization will not be possible if we did not identify and separate each fracture network. This is due to the fact that the effects of fractures are not localizable and are global as it is well known.

Next, we use these local solutions to compute the upscaled equation. Because the local calculations are done in an oversampled domain, the transmissibilities are non-local and extend to the oversampled region, which is $\log (H)$ layers around the target coarse block. Our coarse-grid equations have a similar form to those [14; however, we use different local problems in addition to multicontinua as well as localization. Moreover, we show that one can obtain an accurate solution independent of the contrast and the mesh size. The resulting upscaled equation is written in a discrete form as

$$
\sum_{j, n} T_{m n}^{i, j}\left(u_{n}^{(j)}-u_{m}^{(i)}\right)=q_{m}^{(i)},
$$

where $T_{m n}^{i, j}$ are nonlocal transmissibilities for different continua $m$ and $n$, and $i, j$ correspond to different coarse blocks. We note that $T_{m n}^{i, j}$ are defined in oversampled regions, which are several times larger than the target coarse block. We investigate the non-local dependence of these transmissibilities. We note that the proposed approach modifies the framework developed in [10] to derive the non-local multiple continuum upscaled models.

We note that non-local upscaling is not new in porous media [13, 32, 39, 19, 22, 38, 16 There have been many works related to non-local upscaling, particularly for transport equations. However, even in elliptic equations, one can obtain 
non-local upscaling results. Our proposed method is motivated by the work of Jenny et al., [14, where they derive non-local upscaled models. We would also like to note a recent paper [23, where the authors derive non-local upscaling for problems without high contrast. We remark that the upscaling for flows in fractured media requires multi-continua and thus, to avoid the global upscaling, one needs to take into account the fractures separately and localize their effects. In all these papers, the global formulation of the resulting macroscopic equations is the same with the main difference related to computing upscaled quantities. In this regard, our approach differs from existing works in the literature and address a general case of problems with high contrast and multiple scales.

In the paper, we present some numerical results. In these examples, we compare our proposed upscaled model and the fine-grid models. We compare both averages and downscaled quantities. Our numerical results show that one can achieve a good accuracy with a small localization and using several basis functions per coarse element (continua). More importantly, because the local functions are constants within fractures and the matrix, our variables have physical properties and they denote average pressures in each continua. This is very important for practical simulations as in our previous GMsFEM framework, one needs to extract physical parameters from the variables.

The paper is organized as follows. In the next section, Section 2, we present some preliminaries. In Section 3 and Section 4 , we present our approach. Section 5 is devoted to numerical results. Finally, we present some conclusions.

\section{Preliminaries}

We consider the single-phase flow equation

$$
-\operatorname{div}(\kappa(x) \nabla u)=g, \quad \text { in } \quad D
$$

subject to some boundary conditions. In our numerical examples, we will consider the zero Neumann boundary condition $\nabla u \cdot n=0$. Here, $u$ is the pressure of flow, $g$ is the source term, and $\kappa(x)$ is a heterogeneous field with high contrast. We will be using a variational formulation of (1). To introduce it, we denote by $V=H^{1}(D)$. The variational formulation is to find $u \in V$ such that

$$
\int_{D} \kappa \nabla u \cdot \nabla v=\int_{D} g v
$$

where $g$ satisfies the compatibility condition $\int_{D} g=0$. For the zero Neumann boundary condition, we will use $\int_{D} u=0$ to ensure the well-posedness of the problem.

In this paper, we will mostly focus on applications to fractured media and, for this reason, we also introduce some notations for fractured media. For the fractured media, the domain $D$ can be divided into two sets of regions, that is

$$
D=D_{m} \bigoplus_{i} d_{i} D_{f, i}
$$




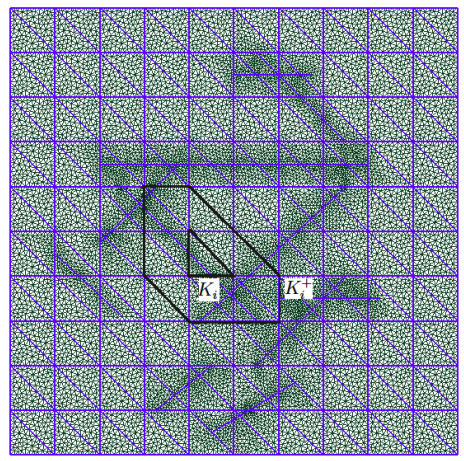

Figure 1: An illustration of coarse and fine mesh in fractured meida. $K_{i}$ denotes a coarse block, $K_{i}^{1}$ denotes one layer oversampling of $K_{i}$.

where $m$ and $f$ corresponds to matrix region and fracture regions respectively, and $d_{i}$ is the aperture of fracture $D_{f, i}$. The permeability in the matrix is $\kappa_{m}$, and the permeability in the $i$-th fracture is denoted by $\kappa_{i}$. We note that the permeabilities of matrix and fractures can differ by orders of magnitude.

The solution of (1) is to find $u \in V$ such that

$$
a(u, v)=(g, v) \quad \forall v \in V,
$$

where $a(u, v)=\int_{D_{m}} \kappa \nabla u \cdot \nabla v+\sum_{i} \int_{D_{f, i}} \kappa_{i} \nabla_{f} u \cdot \nabla_{f} v,(g, v)=\int_{D} g v$.

For the numerical approximation of problem (1), we introduce the notations of fine and coarse grids. We denote by $\mathcal{T}^{H}$ a coarse-grid partition of the domain $D$ with mesh size $H$. By conducting a conforming refinement of the coarse mesh $\mathcal{T}^{H}$, we define a fine mesh $\mathcal{T}^{h}$ of $D$ with mesh size $h$. Typically, we assume that $0<h \ll H<1$, and that the fine-scale mesh $\mathcal{T}^{h}$ is sufficiently fine to fully resolve the small-scale information of the domain, and $\mathcal{T}^{H}$ is a coarse mesh containing many fine-scale features. We let $\left\{K_{i} \mid \quad i=1, \cdots, N\right\}$ be the set of coarse element in $\mathcal{T}^{H}$, where $N$ is the number of coarse blocks. For each $K_{i}$, the oversampled region is denoted by $K_{i}^{+}$, which is an oversampling of $K_{i}$ with a few layers of coarse blocks. An illustration of the fine and coarse meshes, as well as an oversampling region are shown in Figure 1

\section{The non-local multi-continua upscaling}

\subsection{Multi-continua functions}

In this section, we introduce an important part of our method that represents each continua. It will appear as local basis functions representing continua. In general, these functions are automatically computed as described in the next 
section, Section 3.1.1. However, for fractured media, one can use simplified basis functions, which we discuss in Section 3.1.2.

\subsubsection{General spectral setup}

A general setup for identifying a degree of freedom for each continua requires some spectral decomposition. Below, we briefly describe this framework and its relation to special basis functions. We note that this general setup shows the relevance of special basis functions discussed in Section 3.1.2. Moreover, the general setup needs to be used when one can not identify separate fracture networks. As we discussed that the special basis functions do not require any basis computations provided we can identify separate fracture networks.

We first construct a snapshot space $V_{\text {snap }}^{i}$ for each local coarse region $\omega_{i}$ (a coarse neighborhood) or $K_{i}$ (a coarse block). Several choices have been developed for constructing the snapshot basis functions, including (1) the standard fine-scale basis functions, (2) harmonic basis functions which can be obtained by solving the local problems with various boundary conditons, (3) oversampling harmonic basis functions constructed in an oversampled region with standard or randomized boundary conditons, and so on. The offline space $V_{\text {off }}$ is then formed via a dimension reduction in the snapshot space using an auxiliary spectral decomposition. The offline basis obatined represent dominant modes in the snapshot space. In the online stage, one can solve the global problem with various choices of right hand side source term or different boundary conditons.

Here, we present a general example of constructing harmonic snapshots and spectral problem in heterogeneous media. Let $\mathcal{L}$ denotes a linear differential operator, the snapshot basis function is formed by the harmonic extension of fine-grid functions $\delta_{i}(x)$ which are defined on the boudary of local coarse region. That is, a snapshot basis $\psi_{\text {snap }}^{k, i}$ is the solution of

$$
\begin{array}{ccc}
\mathcal{L}\left(\psi_{\text {snap }}^{i, k}\right)=0, & \text { in } \quad S_{i} \\
\psi_{\text {snap }}^{i, k}=\delta_{i, k}, & \text { on } & \partial S_{i}
\end{array}
$$

where $S_{i} \subset D$ denotes the $i$-th local region, i.e., we can take $S_{i}=\omega_{i}$ or $\omega_{i}^{+}$or $K_{i}$ or $K_{i}^{+}$. Note that $\omega_{i}^{+}$and $K_{i}^{+}$represents the oversampled region. The fine grid functions $\delta_{i, k}=\delta_{i}\left(x_{k}\right)$ are defined for all $x_{k} \in \partial S$, where $\left\{x_{k}\right\}$ denote the fine degrees of freedom on the boundary of local coarse region $S_{i}$. The span of these harmonic extensions forms the local snapshot space. One can use randomized boundary conditions to reduce the computational cost associated with snapshot calculations.

Next, we need to design a spectral problem to reduce the dimenson of local multiscale space, and the resulting space can be used as an auxiliary space for further use. Let $V\left(K_{i}\right)$ be the restriction of $V$ on $K_{i}$. Typically, we will find eigenvalues $\lambda_{k}^{i}$ and corresponding eigenfunction $\phi_{\text {off }}^{i, k} \in V\left(K_{i}\right)$ satisfying

$$
a_{i}\left(\phi_{\mathrm{off}}^{i, k}, v\right)=\lambda_{k}^{i} s_{i}\left(\phi_{\mathrm{off}}^{i, k}, v\right), \quad \forall v \in V\left(K_{i}\right),
$$


where bilinear operators $a_{i}$ and $s_{i}$ are defined on $V\left(K_{i}\right) \times V\left(K_{i}\right)$, and they can be symmetric non-negative definite and symmetric positive definite, respectively. For example, for the flow problem in heterogeous medium, one can choose

$$
a_{i}(u, v)=\int_{K_{i}} \nabla u \cdot \nabla v, \quad s_{i}(u, v)=\int_{K_{i}} \tilde{\kappa} u v,
$$

where the definition of $\tilde{\kappa}=\sum_{j} \kappa\left|\nabla \chi_{j}\right|^{2}$ is motivated by the analysis, and $\nabla \chi_{j}$ denotes the multiscale partition of unity function. We arrange the eigenvalues of (4) ascendingly, and then select the first $l_{i}$ eigenfunctions corresponding to the small eigenvalues to construct the offline basis functions. The span of these multiscale basis functions will form an auxiliary space, $V_{\text {aux }}^{(i)}:=\operatorname{span}\left\{\phi_{\text {off }}^{i, k}, \quad 1 \leq\right.$ $\left.k \leq l_{i}\right\}$, where $1 \leq i \leq N$ and $N$ is the number of coarse blocks. We note that the auxiliary space needs to be chosen appropriately, that is, all basis functions corresponding to small eigenvalues (representing the channels) have to be included in the space.

At this point, we can construct the multiscale basis $\psi_{j, m s}^{(i)}$ using the auxiliary space $V_{\text {aux }}^{(i)}$ by ensuring the constraint energy minimization (see [10] for details). We let $I_{i}$ be the index set containing all coarse block indices $\ell$ with $K_{\ell} \subset K_{i}^{+}$. To construct the required basis, we find $\psi_{j, m s}^{(i)}$ by solving

$$
\begin{aligned}
\sum_{\ell \in I_{i}} a_{\ell}\left(\psi_{j, m s}^{(i)}, w\right)+\sum_{\ell \in I_{i}} s_{\ell}(w, \mu) & =0, \quad \forall w \in V_{0}\left(K_{i}^{+}\right), \\
s_{\ell}\left(\psi_{j, m s}^{(i)}, \nu\right) & =s_{\ell}\left(\phi_{\mathrm{off}}^{i, j}, \nu\right), \quad \forall \nu \in V_{\mathrm{aux}}^{(\ell)}, \forall \ell \in I_{i},
\end{aligned}
$$

where $\phi_{\text {off }}^{i, j} \in V_{\text {aux }}^{(i)}$ is a basis in auxiliary space and $V_{0}\left(K_{i}^{+}\right)=H_{0}^{1}\left(K_{i}^{+}\right)$. The

contraint basis obtained form the multiscale space $V_{m s}:=\operatorname{span}\left\{\psi_{j, m s}^{(i)}, \quad 1 \leq\right.$ $\left.j \leq l_{i}, 1 \leq i \leq N\right\}$, which will be used for find the multiscale solution. Since the auxiliary space contains basis functions which capture the high-contrast features, it has been proved that the convergence of the this method is independent of the contrast and the convergence rate is in order of the coarse mesh size for appropriate oversampling size. We remark that this framework is general and can work for complex heterogeneities and multi-continuum case. For a simplified case, for example, when the fracture networks are known, we can construct some simplified basis functions with constraint energy minimization. The details are presented in the next section.

\subsubsection{Simplified basis functions representing continua}

In this section, we discuss the construction of simplified basis for fractured media. Our approach is motivated by the Constraint Energy Minimizing GMsFEM method proposed in [10, where oversampling regions are used to compute the multiscale basis functions. We aim to construct simplified basis which has spatial decay property and can separate each contina automatically. With these simplified basis, non-local (restricted to oversampled regions) transfer and effective properties can be constructed. 
The main idea behind this construction is to use constants within each separate fracture network within each coarse block and a constant for each matrix. This simplified construction of auxiliary space uses minimal degrees of freedom in each continua. As a result, we will obtain an upscaled equation with a minimal size. A major drawback of our construction is that it assumes that we know the separate fracture networks and assign a constant. Some of physical applications can identify separate fracture networks and thus our assumption is valid for many cases. Next, we present a detailed description of basis construction.

We start by defining a simplified auxiliary space. Consider an oversampling region $K_{i}^{+}$of coarse block $K_{i}$, we write $F^{(j)}=\left\{f_{m}^{(j)} \mid f_{m}^{(j)}=D_{f, m} \cap K_{j} \neq \varnothing\right\}$ as the set of discrete fractures inside any coarse element $K_{j} \subset K_{i}^{+}$, and let $L_{j}=\operatorname{dim}\left\{F^{(j)}\right\}$. Let $\phi_{0}^{(i)}, \phi_{l}^{(i)}\left(l=1, \cdots, L_{i} ; i=1, \cdots, N\right)$ satisfy the following conditions

$$
\begin{aligned}
& \int_{K_{j}} \phi_{0}^{(i)}=\delta_{i j}, \quad \int_{f_{m}^{(j)}} \phi_{0}^{(i)}=0 \\
& \int_{K_{j}} \phi_{l}^{(i)}=0, \quad \int_{f_{m}^{(j)}} \phi_{l}^{(i)}=\delta_{i j} \delta_{m l} .
\end{aligned}
$$

The number of fracture continuum in the coarse block $K_{i}$ is denoted by $L_{i}$. We can see that, $\phi_{0}^{(i)}$ has average 1 in the matrix continua of coarse element $K_{i}$, and it has average 0 in other coarse blocks $K_{j} \subset K_{i}^{+}$as well as any fracture inside $K_{i}^{+}$. As for $\phi_{l}^{(i)}$, it has average 1 on the $l$-th fracture continua inside the coarse element $K_{i}$, and average 0 in other fracture continua as well as the matrix continua of any coarse block $K_{j} \subset K_{i}^{+}$. The auxiliary space is then $V_{\mathrm{aux}}^{(i)}=\operatorname{span}\left\{\phi_{l}^{(i)}, \quad 0 \leq l \leq L_{i}\right\}$. We note this definition separates the matrix and fractures, and each basis represents a continua.

Define the subspace $V_{1}\left(K_{i}^{+}\right):=\left\{v \in V_{0}\left(K_{i}^{+}\right) \mid \int_{K} v=0, \int_{f_{m}^{(j)}} v=0, \forall K \subset\right.$ $\left.K_{i}^{+}, \forall j, 1 \leq m \leq L_{j}\right\}$. Let $G_{l o c}^{(i)}: V \rightarrow V_{1}\left(K_{i}^{+}\right)$be a localized operator such that

$$
a\left(G_{l o c}^{(i)}(u), v\right)=a(u, v), \quad \forall v \in V_{1}\left(K_{i}^{+}\right) .
$$

where $V_{0}\left(K_{i}^{+}\right)=\left\{v \in V\left(K_{i}^{+}\right) \mid v=0\right.$ on $\left.\partial K_{i}^{+}\right\}$, and $V\left(K_{i}^{+}\right)$is the fine grid space over an oversampled region $K_{i}^{+}$. We define $\psi_{m}^{(i)}:=\phi_{m}^{(i)}-G_{l o c}\left(\phi_{m}^{(i)}\right)$, and note that the multiscale basis $\psi_{m}^{(i)}$ allows a spatial decay. In order to ensure constraint energy minimizing property, $\psi_{m}^{(i)}$ are constructed by solving the following local problem on the fine grid

$$
\begin{aligned}
& a\left(\psi_{m}^{(i)}, v\right)+\sum_{K_{j} \subset K_{i}^{+}}\left(\mu_{0}^{(j)} \int_{K_{j}} v+\sum_{m \leq L_{j}} \mu_{m}^{(j)} \int_{f_{m}^{(j)}} v\right)=0, \quad \forall v \in V_{0}\left(K_{i}^{+}\right), \\
& \int_{K_{j}} \psi_{m}^{(i)}=\delta_{i j} \delta_{0 m}, \quad \forall K_{j} \subset K_{i}^{+}, \\
& \int_{f_{m}^{(j)}} \psi_{m}^{(i)}=\delta_{i j} \delta_{n m}, \quad \forall f_{m}^{(j)} \in F^{(j)}, \forall K_{j} \subset K_{i}^{+} .
\end{aligned}
$$



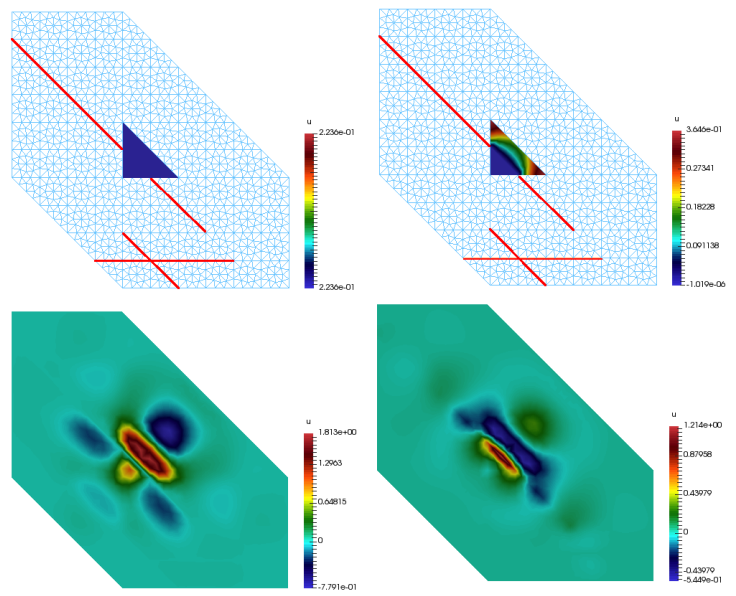

Figure 2: Top: spectral eigenfunctions. Bottom: simplified basis.

Finally, the multiscale space for fractured media is $V_{m s}=\operatorname{span}\left\{\psi_{m}^{(i)}, \quad 0 \leq m \leq\right.$ $\left.L_{i}, 1 \leq i \leq N\right\}$.

In Figure 2, we present a comparison between eigenfunctions constructed in Section 3.1.1, and the simplified basis constructed in this secion for a same coarse block $K$. We note that the support of the eigenbasis is in $K$, and the support of the simplified basis the oversampled region $K^{+}$. We can see from the top of Figure 2 that, the first eigenfunction is constant in the coarse block $K$, and the second eigenfunction is constant on the fracture within coarse block $K$. For the simplified basis at the bottom of Figure 2. we observe that the first basis represent the matrix, and the second one represent the fracture. This indicates the relation between our simplified basis and the eigenfunctions obtained from the spectral problem. Again, we note that the simplified functions assume that one knows separate fracture networks and uses minimal degrees of freedom to setup a coarse system, which represent the average pressures.

\subsection{Transmissibility computations}

\subsubsection{General spectral basis}

In the general heterogeneous case, we have constructed the constaint energy minimization basis $\left\{\psi_{m, m s}^{(i)}, \quad 1 \leq m \leq l_{i}, 1 \leq i \leq N\right\}$ as shown in (5). The transmissibility matrix can be constructed by calculating

$$
T_{m n, l o c}^{(i, j)}=a\left(\psi_{m, m s}^{(i)}, \psi_{n, m s}^{(j)}\right),
$$

where $m, n$ denote the $m$ - or $n$-th basis in a coarse block, $i, j$ denote the indices of coarse blocks. 


\subsubsection{Simplified basis}

For the fractured media, where we assume the fractured networks are known, we constructed the simplified basis $\left\{\psi_{m}^{(i)}, \quad 0 \leq m \leq L_{i}, 1 \leq i \leq N\right\}$ by solving (6). We define $T_{l o c}$ by

$$
T_{m n, l o c}^{(i, j)}=a\left(\psi_{m}^{(i)}, \psi_{n}^{(j)}\right) .
$$

We note that $m, n$ denotes different continua, and $i, j$ are the indices for coarse blocks. This construction shows that we can get non-local (in the oversampled regions) transfer and effective properties for multi-continuum.

\subsection{Approximation using local multiscale basis}

Using the transmissibility defined in (7) and (8), our problem is to find the approximation solution $\overrightarrow{u_{T}}$ such that

$$
\sum_{n} \sum_{j} T_{m n, l o c}^{(i, j)}\left(\left[u_{T}\right]_{n}^{(j)}-\left[u_{T}\right]_{m}^{(i)}\right)=g_{m}^{(i)}
$$

With a simplification of indices, we write $T_{l o c}$ in the following form

$$
\left[\begin{array}{cccc}
t_{11} & t_{12} & \cdots & t_{1 n} \\
t_{21} & t_{22} & \cdots & t_{2 n} \\
\vdots & \vdots & \ddots & \vdots \\
t_{n 1} & t_{n 2} & \cdots & t_{n n}
\end{array}\right]
$$

where $n=\sum_{i=1}^{N}\left(1+L_{i}\right)$, and $1+L_{i}$ means the one matrix continua plus the number of discrete fractures in coarse block $K_{i}$, and $N$ is the number of coarse blocks. The system (9) can then be expressed as in the matrix form

$$
A_{T} \cdot \overrightarrow{u_{T}}=\left(\begin{array}{cccc}
-\sum_{j} t_{1 j} & t_{12} & \cdots & t_{1 n} \\
t_{21} & -\sum_{j} t_{2 j} & \cdots & t_{2 n} \\
\vdots & \vdots & \ddots & \vdots \\
t_{n 1} & t_{n 2} & \cdots & -\sum_{j} t_{n j}
\end{array}\right)\left(\begin{array}{c}
{\left[u_{T}\right]_{0}^{(1)}} \\
{\left[u_{T}\right]_{1}^{(1)}} \\
\vdots \\
{\left[u_{T}\right]_{N}^{\left(L_{N}\right)}}
\end{array}\right)=\left(\begin{array}{c}
g_{0}^{(1)} \\
g_{1}^{(1)} \\
\vdots \\
g_{N}^{\left(L_{N}\right)}
\end{array}\right)
$$

We remark that the summation of each row in $A_{T}$ is zero, which ensures the mass conservation.

\section{Time-dependent problem}

We also consider the time-dependent single-phase flow and use spatial upscaling derived above. In particular, we consider

$$
\frac{\partial u}{\partial t}-\operatorname{div}(\kappa \nabla u)=g, \quad \text { in } \quad D .
$$


The fine scale solution can be found using the standard finite element scheme, with backward Euler method for time discretization:

$$
\left(\frac{u^{n}-u^{n-1}}{d t}, v\right)+\left(\kappa \nabla u^{n}, \nabla v\right)=(g, v) .
$$

In matrix form, we have

$$
M_{f} u^{n}+A_{f} u^{n}=b_{f}+M_{f} u^{n-1},
$$

where $M_{f}$ and $A_{f}$ are fine scale mass and stiffness matrix respectively, $b_{f}$ is the right hand side vector.

For the coarse scale approximation, we will solve

$$
M_{T} u_{T}^{n}+A_{T} u_{T}^{n}=b_{f}+M_{T} u_{T}^{n-1},
$$

where $A_{T}$ is defined in (11) and $M_{T}$ is an approximation of coarse scale mass matrix. We note that both $A_{T}$ and $M_{T}$ are non-local and defined for each continua. One can write the non-local upscaled equation as

$$
\sum_{j, n} M_{m n}^{i, j} \frac{d}{d t} u_{m}^{(i)}+\sum_{j, n} T_{m n}^{i, j}\left(u_{n}^{(j)}-u_{m}^{(i)}\right)=g_{m}^{(i)}
$$

\section{$5 \quad$ Numerical results}

\subsection{Steady state case}

In this section, we present some representative numerical examples. We plan to consider more realistic and complicated fracture systems in our future works.

In this example, we use the fractured media as shown in the left of Figure 3 The permeability of the matrix is $\kappa_{m}=1$, and the permeability of the fractures are $\kappa_{f}=10^{2}$. The source term in the right hand side of the equation is piecewise constant functions with $f=10^{2}$ for $0 \leq x \leq 0.2,0.3 \leq y \leq 0.4$, and $f=-10^{2}$ for $0 \leq x \leq 0.2,0.7 \leq y \leq 0.8$.

The degrees of freedom for fine-scale approximation are 22642 . Let $u_{f}$ be the fine scale solution. We define the average of fine-scale solution $\bar{u}$ such that $\left.\bar{u}\right|_{K_{i}}=$ $\int_{K_{i}} u,\left.\bar{u}\right|_{f_{m}^{(i)}}=\int_{f_{m}^{(i)}} u$. These are the average pressures, which are computed with our approach. We plot $u$ and $\bar{u}$ in Figure 4 .

For the coarse scale approximation, we take the coarse mesh size as $H=$ $1 / 10,1 / 20$ respectively for numerical simulations. When $H=1 / 10$, the coarse scale degrees of freedom is 282 . When $H=1 / 20$, the coarse scale degrees of freedom is 927 .

First, we present the local solutions constructed. We take the example for the coarse mesh with $H=1 / 20$ and consider a coarse block $K_{i}$ with one discrete fracture in it. The two local solutions are shown in Figure 5 when we use two oversampling layers, and in Figure 6 when we use six oversampling layers, respectively. From the figures, we notice that with two layers of oversampling, 

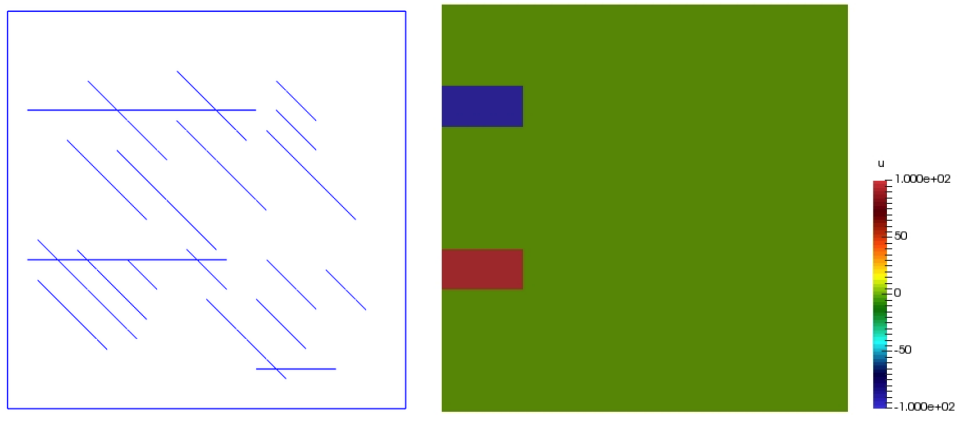

Figure 3: Left: A fractured meida. Right: source term.
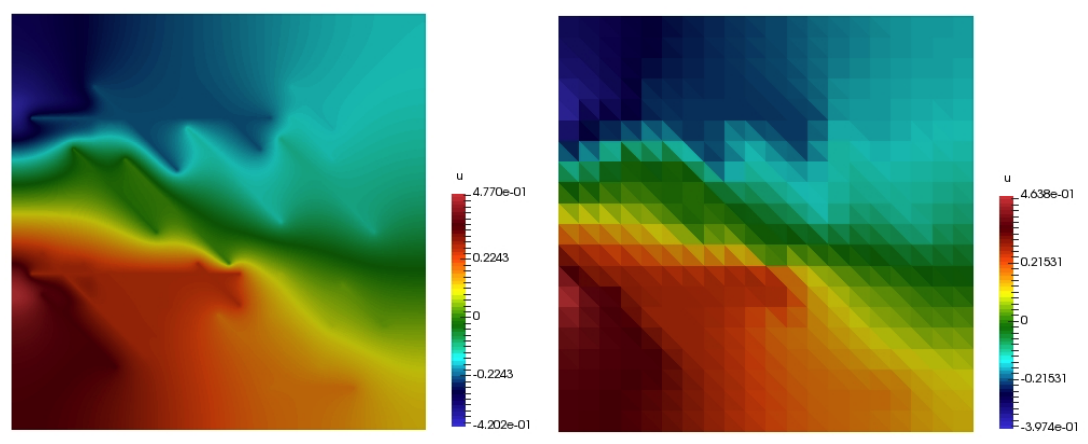

Figure 4: Left: fine scale solution. Rght: average of fine scale solution.
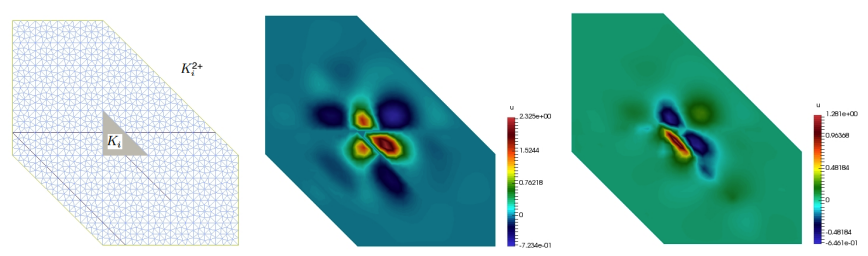

Figure 5: From left to right: A coarse block $K_{i}$ with two oversampling layers $K_{i}^{2+}$. Local solution w.r.t matrix. Local solution w.r.t. the fracture. 

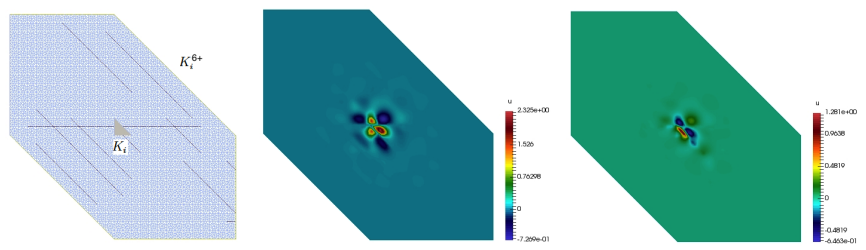

Figure 6: From left to right: A coarse blcok $K_{i}$ with six oversampling layers $K_{i}^{6+}$. Local solution w.r.t matrix. Local solution w.r.t. the fracture.

the local distribution has a decay property, and the local solution almost vanish outside the oversampling region with six layers. This indicates that one can localize the effects.

We present the error $\left\|u_{T}-\bar{u}\right\|_{L_{2}}$ in Tables 1 and 2 for $H=1 / 10$ and $H=$ $1 / 20$, respectively. From the numerial results, we observe a good convergence comparing $u_{T}$ with the averaged fine-scale solution. It can be seen that, with 2 layers of oversampling, the error $\left\|u_{T}-\bar{u}\right\|_{L_{2}}$ is $1.46 \%$ for $H=1 / 10$. For the case $H=1 / 20,4$ layers of oversampling gives an error of $0.008 \%$. This indicates the upscaled equation in our modified method can use small local regions. We plot the upscaled solutions using different size of oversampling region and compare them with the averaged fine-scale solution for the case $H=1 / 20$. The results are presented in Figure 7. It shows that we can obtain very good accuracy with 4 layers of oversampling.

\begin{tabular}{|c|c|}
\hline Oversampling & $\left\|u_{T}-\bar{u}\right\|_{L_{2}}(\%)$ \\
\hline 1 & 21.97 \\
\hline 2 & 1.46 \\
\hline 3 & 0.015 \\
\hline 4 & 0.0008 \\
\hline global & $4.57 \mathrm{e}-10$ \\
\hline
\end{tabular}

Table 1: Coarse mesh size 1/10. Upscaling errors when oversampling with $1,2,3,4$ layers of coarse blocks. Last row shows the error when using global domain for the local computations.

Next, we present numerical results for the transmissibility matrix $T_{l o c}$. We display $T_{m n, l o c}^{(i, j)}$ for two different coarse blocks $K_{i}$ in Figure 8 and Figure 10 in the global domain. In the left of these two figures, we plot the transmissibility between the element $K_{i}$ and its neighboring elements for the matrix continua. In the right of the figures, we show the transmissibility for the fracture continua between $K_{i}$ and neighboring elements. We notice that the regions of influence are almost within the 4 layers of overampling region, which is in accordance with our numerical results. Figure 9 shows a one dimensional plot of transmissibility between an element $K_{i}$ and other coarse elements in the region marked by 

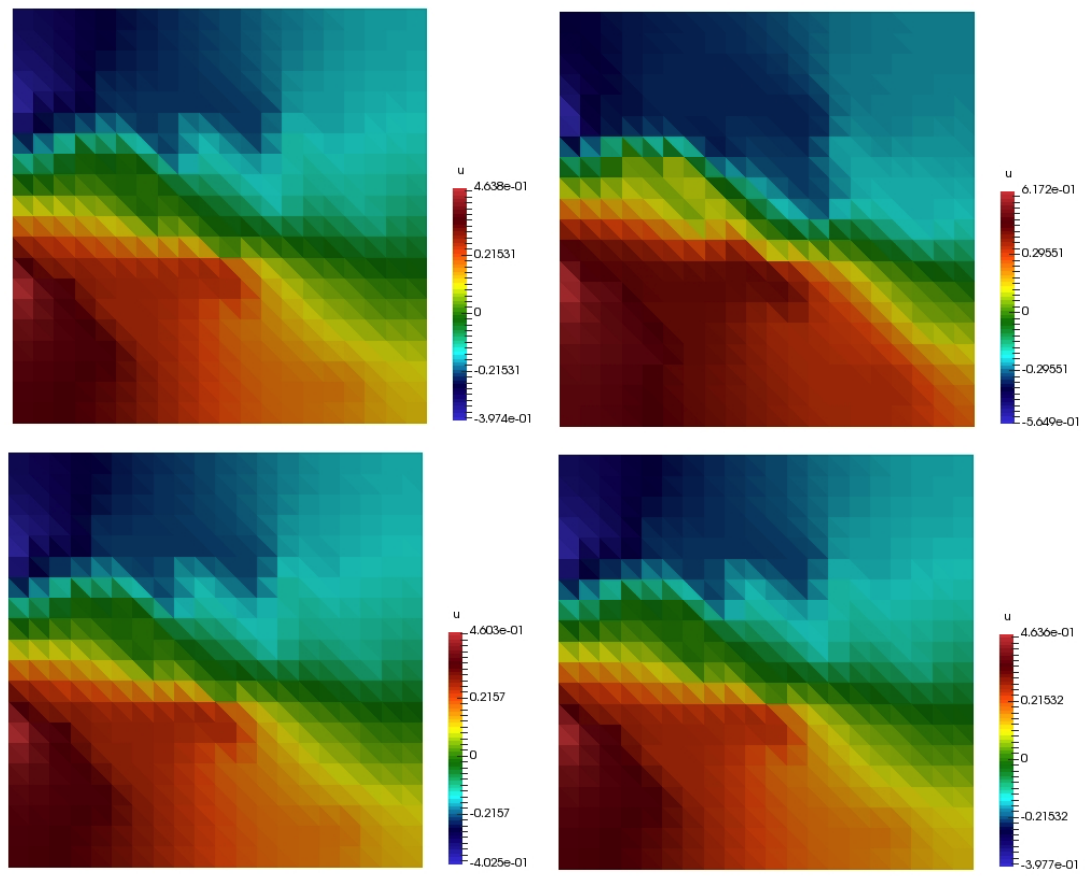

Figure 7: Corse mesh size 1/20. Upper left: coarse scale solution using global domain for local computations. Upper right: coarse scale solution with oversampling size 1. Lower left: coarse scale solution with oversampling size 2. Lower right: coarse scale solution with oversampling size 4 . 


\begin{tabular}{|c|c|}
\hline Oversampling & $\left\|u_{T}-\bar{u}\right\|_{L_{2}}(\%)$ \\
\hline 1 & 40.63 \\
\hline 2 & 22.95 \\
\hline 4 & 0.008 \\
\hline 6 & 0.0007 \\
\hline global & $6 \mathrm{e}-5$ \\
\hline
\end{tabular}

Table 2: Coarse mesh size 1/20. Upscaling errors when oversampling with $1,2,4,6$ layers of coarse blocks. Last row shows the error when using global domain for local computations.
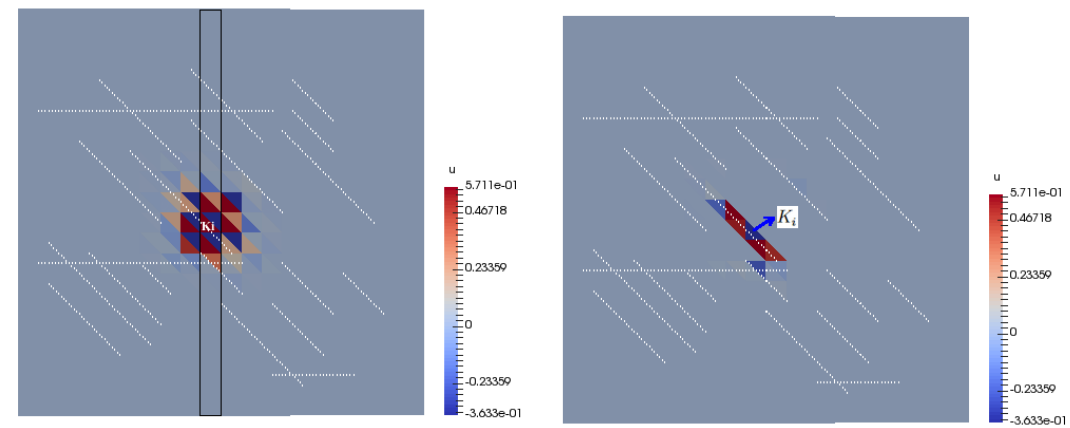

Figure 8: Using global domain for local computation. Left: Transmissibility between an element $K_{i}$ and neighboring elements for matrix. Right: Transmissibility between $K_{i}$ and neighboring elements for fractures. The dotted white lines denotes fractures in the domain.

the black box in Figure 8 (left). We remark that the transmissibility matrix computed using global domain is exact, which can be used as a reference. From the subplot on the top of Figure 9, we note that the transmissibility between $K_{i}$ and other coarse blocks along the slab decays fast. It can be seen from the lognormal plot on the bottom of Figure 9 , that with one layer of oversampling, the values in the transmissibility matrix has large errors, however, with four layers of oversampling, the transmissibility is quite accurate.

\subsection{Time-dependent case}

In this example, we present numerical results for the time-dependent case. The source term and geometry are the same as in the previous section, shown in Figure 3. The simulation runs for a total time of $T=1.0$, we present the error between the coarse scale solution and average fine-scale solution at time instances $t=0.1,0.5$ and 1.0. The results show good accuracy of the proposed method. 

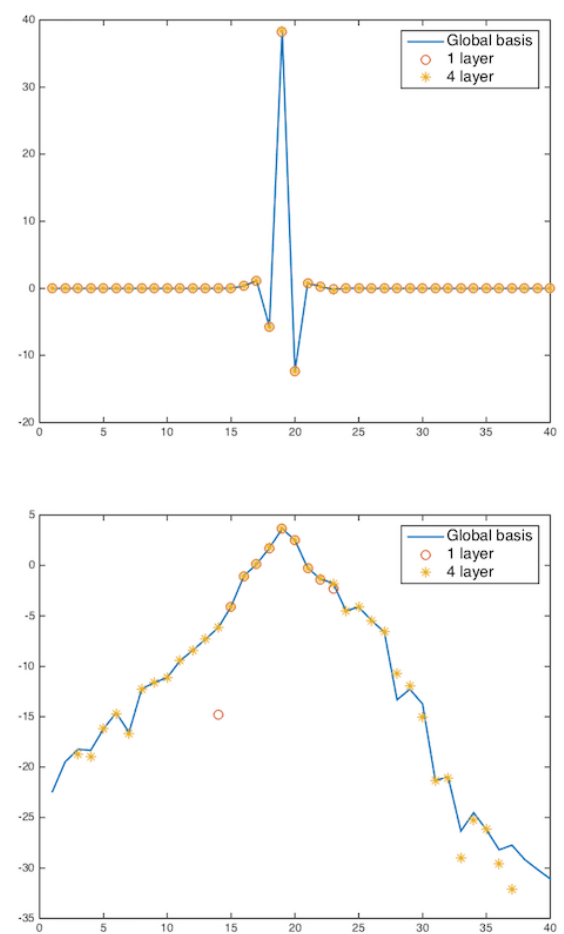

Figure 9: Transmissibility between an element $K_{i}$ and elements in the cross section marked by the black box in Figure 8 (left). Top: plot of exact values. Bottom: log plot of absolute values.
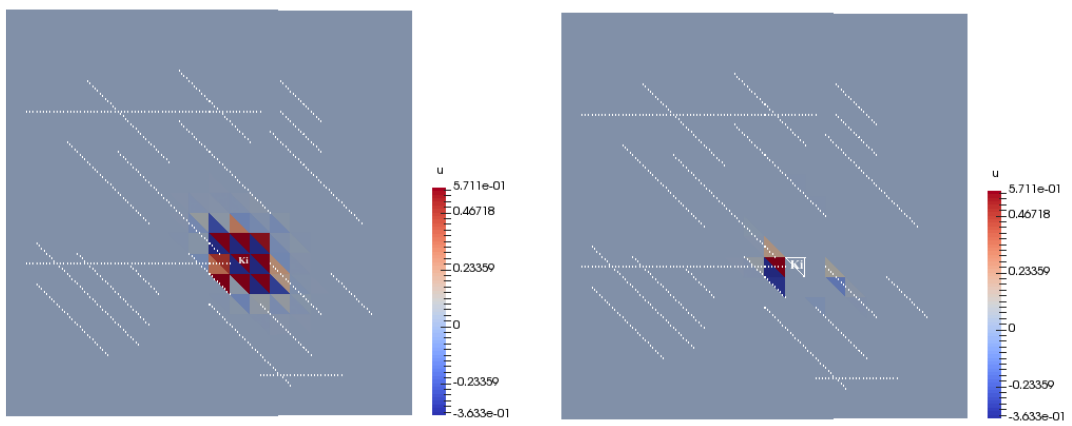

Figure 10: Using the global domain for local computations. Left: Transmissibility between an element $K_{i}$ and neighboring elements for matrix. Right: Transmissibility between $K_{i}$ and neighboring elements for fractures. The dotted white lines denotes fractures in the domain. 


\begin{tabular}{|c|c|c|c|}
\hline Oversampling & $t=0.1$ & $t=0.5$ & $t=1.0$ \\
\hline 1 & 40.055 & 61.027 & 62.741 \\
\hline 2 & 1.065 & 1.035 & 1.035 \\
\hline 4 & 0.076 & 0.009 & 0.008 \\
\hline 6 & 0.072 & 0.002 & 0.0007 \\
\hline global & 0.072 & 0.001 & 0.0006 \\
\hline
\end{tabular}

Table 3: Error $\left\|u_{T}-\bar{u}\right\|_{L_{2}}(\%)$. Coarse mesh size 1/20. Upscaling errors when oversampling with 1,2,4,6 layers of coarse blocks. Last row shows the error when using global domain for local computation.

\section{Conclusions}

We propose a non-local upscaling framework based on some recently developed multiscale methods [10. The approach uses constraints local solutions to compute the non-local effective transmissibilities for each continua restricted to the oversampled regions, which are several times larger than the target coarse block. The continua are defined by choosing piecewise constant functions for each fracture network and the matrix. Because of our choices of the variables, the resulting system defined average pressures for each continua, which is important for applications. The resulting nonlocal upscaled equation is defined in a small neighborhood of the coarse block. The local problems for the computation of effective properties are formulated for each continua such that it is orthogonal to other continua defined by piecewise constant functions. We note that the use of non-local upscaled model for porous media flows is not new, e.g., in [14, the authors derive non-local approach. One can use these ideas to model non-local upscaled quantities analytically, which we will pursue in our future works. We present numerical results, which show that the proposed approach can provide a good accuracy. We compare average pressures and study the decay property of local upscaled quantities. Our numerical examples are simplistic and we plan to consider more general fracture systems in our future works.

\section{Acknowledgement}

The research of Eric Chung is partially supported by the Hong Kong RGC General Research Fund (Project 14317516) and the CUHK Direct Grant for Research 2016-17. MV's work is partially supported by Mega-grant of the Russian Federation Government (N 14.Y26.31.0013). YE would like to thank Lou Durlofsky for the discussions related to fracture-matrix upscaling during IPAM meeting. 

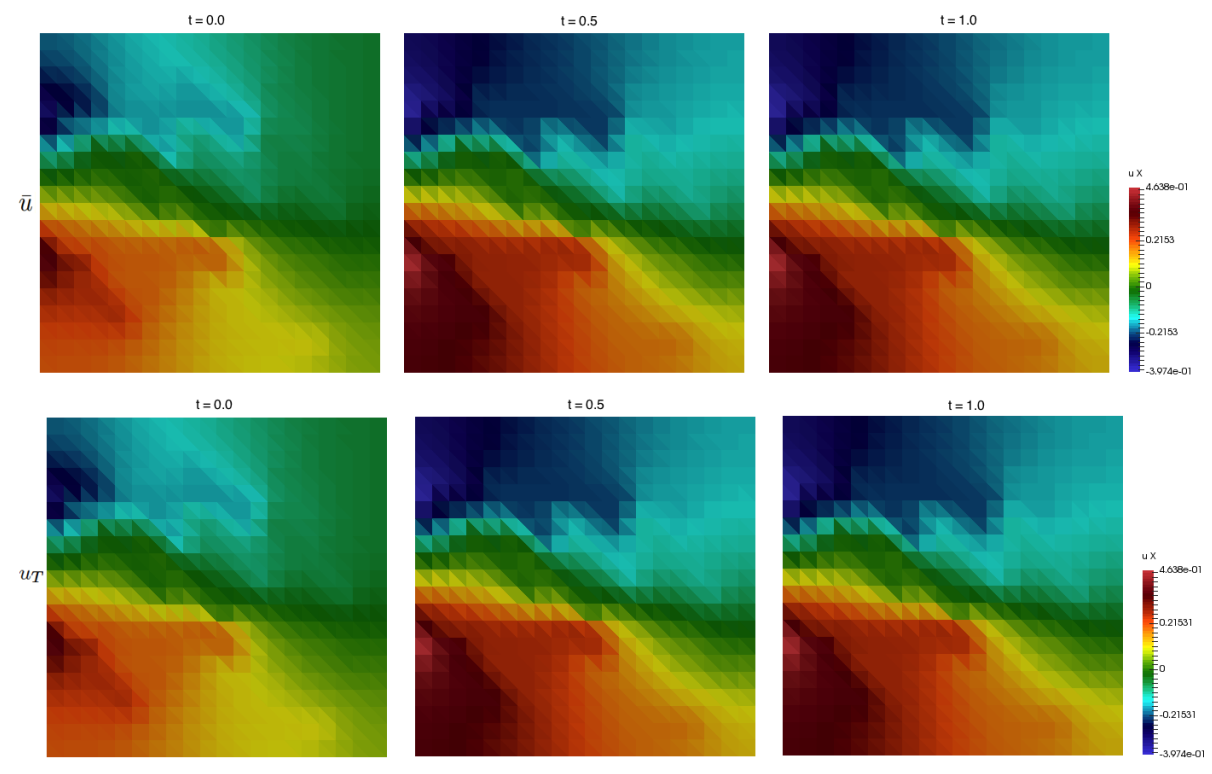

Figure 11: Oversampling size 4. Top: Average of fine scale solution at $t=$ $0,0.5,1.0$. Bottom: Coarse scale solution at $t=0,0.5,1.0$.

\section{References}

[1] T. Arbogast, G. Pencheva, M.F. Wheeler, and I. Yotov. A multiscale mortar mixed finite element method. SIAM J. Multiscale Modeling and Simulation, 6(1):319-346, 2007.

[2] A. Bourgeat. Homogenized behavior of two-phase flows in naturally fractured reservoirs with uniform fractures distribution. Comp. Meth. Appl. Mech. Engrg., 47:205-215, 1984.

[3] F. Brezzi, L. P. Franca, T. J. R. Hughes, and A. Russo. $b=\int$ g. Comput. Methods in Appl. Mech. and Engrg., 145:329-339, 1997.

[4] Y. Chen, L. Durlofsky, M. Gerritsen, and X. Wen. A coupled local-global upscaling approach for simulating flow in highly heterogeneous formations. Advances in Water Resources, 26:1041-1060, 2003.

[5] Z. Chen and T.Y. Hou. A mixed multiscale finite element method for elliptic problems with oscillating coefficients. Mathematics of Computation, 72:541-576, 2002.

[6] C.C. Chu, I.G. Graham, and T. Hou. A new multiscale finite element methods for high-contrast elliptic interface problem. Mathematics of Computation, 79:1915-1955, 2010. 
[7] Eric Chung, Yalchin Efendiev, and Thomas Y Hou. Adaptive multiscale model reduction with generalized multiscale finite element methods. Journal of Computational Physics, 320:69-95, 2016.

[8] Eric T Chung, Yalchin Efendiev, Tat Leung, and Maria Vasilyeva. Coupling of multiscale and multi-continuum approaches. GEM-International Journal on Geomathematics, 8(1):9-41, 2017.

[9] Eric T Chung, Yalchin Efendiev, and Wing Tat Leung. Generalized multiscale finite element methods for wave propagation in heterogeneous media. Multiscale Modeling \& Simulation, 12(4):1691-1721, 2014.

[10] Eric T Chung, Yalchin Efendiev, and Wing Tat Leung. Constraint energy minimizing generalized multiscale finite element method. arXiv preprint arXiv:1704.03193, 2017.

[11] Davide Cortinovis and Patrick Jenny. Iterative galerkin-enriched multiscale finite-volume method. Journal of Computational Physics, 277:248267, 2014.

[12] M. Cruz and A. Petera. A parallel monte-carlo finite element procedure for the analysis of multicomponent random media. Int. J. Numer. Methods Engrg., 38:1087-1121, 1995.

[13] John H Cushman, Lynn S Bennethum, and Bill X Hu. A primer on upscaling tools for porous media. Advances in Water Resources, 25(8):1043-1067, 2002 .

[14] Amir H Delgoshaie, Daniel W Meyer, Patrick Jenny, and Hamdi A Tchelepi. Non-local formulation for multiscale flow in porous media. Journal of Hydrology, 531:649-654, 2015.

[15] L.J. Durlofsky. Numerical calculation of equivalent grid block permeability tensors for heterogeneous porous media. Water Resour. Res., 27:699-708, 1991.

[16] LJ Durlofsky, Y Efendiev, and V Ginting. An adaptive local-global multiscale finite volume element method for two-phase flow simulations. Advances in Water Resources, 30(3):576-588, 2007.

[17] Louis J Durlofsky. Upscaling of geocellular models for reservoir flow simulation: a review of recent progress. In 7th International Forum on Reservoir Simulation Bühl/Baden-Baden, Germany, pages 23-27. Citeseer, 2003.

[18] B. Dykaar and P. K. Kitanidis. Determination of the effective hydraulic conductivity for heterogeneous porous media using a numerical spectral approach: 1. method. Water Resour. Res., 28:1155-1166, 1992.

[19] Y Efendiev and LJ Durlofsky. A generalized convection-diffusion model for subgrid transport in porous media. Multiscale Modeling 85 Simulation, 1(3):504-526, 2003. 
[20] Y. Efendiev, J. Galvis, and T. Hou. Generalized multiscale finite element methods. Journal of Computational Physics, 251:116-135, 2013.

[21] Y. Efendiev and T. Hou. Multiscale Finite Element Methods: Theory and Applications. Springer, 2009.

[22] Yalchin Efendiev, LJ Durlofsky, and SH Lee. Modeling of subgrid effects in coarse-scale simulations of transport in heterogeneous porous media. Water Resources Research, 36(8):2031-2041, 2000.

[23] Dietmar Gallistl and Daniel Peterseim. Computation of local and quasilocal effective diffusion tensors in elliptic homogenization. arXiv preprint arXiv:1608.02092, 2016.

[24] H. Hajibeygi, D. Karvounis, and P. Jenny. A loosely coupled hierarchical fracture model for the iterative multiscale finite volume method. Society of Petroleum Engineers. doi:10.2118/141991-MS.

[25] H. Hajibeygi, D. Kavounis, and P. Jenny. A hierarchical fracture model for the iterative multiscale finite volume method. Journal of Computational Physics, 230(4):8729-8743, 2011.

[26] Hadi Hajibeygi, Dimitris Karvounis, Patrick Jenny, et al. A loosely coupled hierarchical fracture model for the iterative multiscale finite volume method. In SPE Reservoir Simulation Symposium. Society of Petroleum Engineers, 2011.

[27] T. Hou and X.H. Wu. A multiscale finite element method for elliptic problems in composite materials and porous media. J. Comput. Phys., 134:169189, 1997.

[28] T.J.R. Hughes. Multiscale phenomena: Green's functions, the dirichletto-neumann formulation, subgrid scale models, bubbles and the origins of stabilized methods. Comput. Methods Appl. Mech Engrg., 127:387-401, 1995.

[29] T.J.R. Hughes, G.R. Feijóo, L. Mazzei, and J.-B. Quincy. The variational multiscale method - a paradigm for computational mechanics. Comput. Methods Appl. Mech Engrg., 127:3-24, 1998.

[30] P. Jenny, S.H. Lee, and H. Tchelepi. Multi-scale finite volume method for elliptic problems in subsurface flow simulation. J. Comput. Phys., 187:4767, 2003.

[31] R. Juanes and T. W. Patzek. A variational multiscale finite element method for multiphase flow in porous media. Finite Elements in Analysis and Design, 41(7-8):763-777, 2005. 
[32] Persefoni E Kechagia, Ioannis N Tsimpanogiannis, Yanis C Yortsos, and Peter C Lichtner. On the upscaling of reaction-transport processes in porous media with fast or finite kinetics. Chemical engineering science, 57(13):2565-2577, 2002.

[33] I. Lunati and P. Jenny. Multi-scale finite-volume method for highly heterogeneous porous media with shale layers. In Proceedings of the 9th European Conference on the Mathematics of Oil Recovery (ECMOR), Cannes, France, 2004.

[34] Axel Målqvist and Daniel Peterseim. Localization of elliptic multiscale problems. Mathematics of Computation, 83(290):2583-2603, 2014.

[35] J. Nolen, G. Papanicolaou, and O. Pironneau. A framework for adaptive multiscale method for elliptic problems. SIAM J. Multiscale Modeling and Simulation, 7:171-196, 2008.

[36] Diego Paredes, Frédéric Valentin, and Rodolfo Araya. Two-level multiscale hybrid method for elliptic problems. In Congress on Numerical Methods in Engineering CMN2017, volume 3, page 5, 2017.

[37] M. Peszyńska, M. Wheeler, and I. Yotov. Mortar upscaling for multiphase flow in porous media. Comput. Geosci., 6(1):73-100, 2002.

[38] TC Wallstrom, MA Christie, LJ Durlofsky, and DH Sharp. Effective flux boundary conditions for upscaling porous media equations. Transport in Porous Media, 46(2):139-153, 2002.

[39] Xian-Huan Wen and J Jaime Gómez-Hernández. Upscaling hydraulic conductivities in heterogeneous media: An overview. Journal of Hydrology, 183(1):ix-xxxii, 1996.

[40] X.H. Wu, Y. Efendiev, and T.Y. Hou. Analysis of upscaling absolute permeability. Discrete and Continuous Dynamical Systems, Series B., 2:158-204, 2002 . 